\title{
FAECAL INCONTINENCE
}

\author{
E S Kiff
}

\section{People are recluctant to discuss faecal incontinence. There is no "polite" vocabulary.}

Faecal incontinence is extremely distressing. In the past those afflicted often suffered in silence rather than face the embarrassment of discussing the problem with a doctor. Happily, today more is known about the condition so that in many cases it can be totally alleviated or substantially improved.

\section{Aetiology}

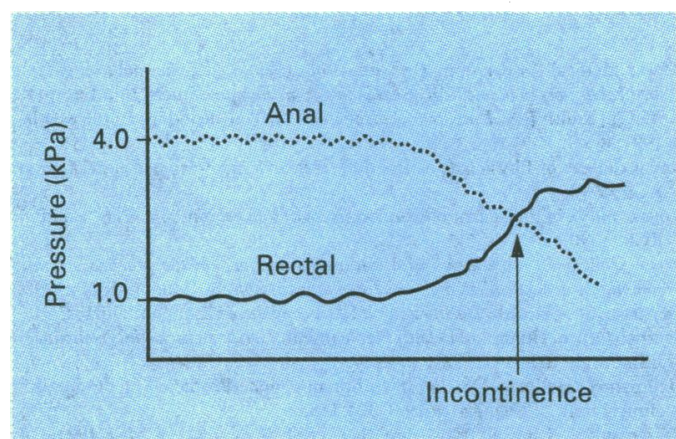

Faecal incontinence occurs when rectal pressure exceeds anal pressure.

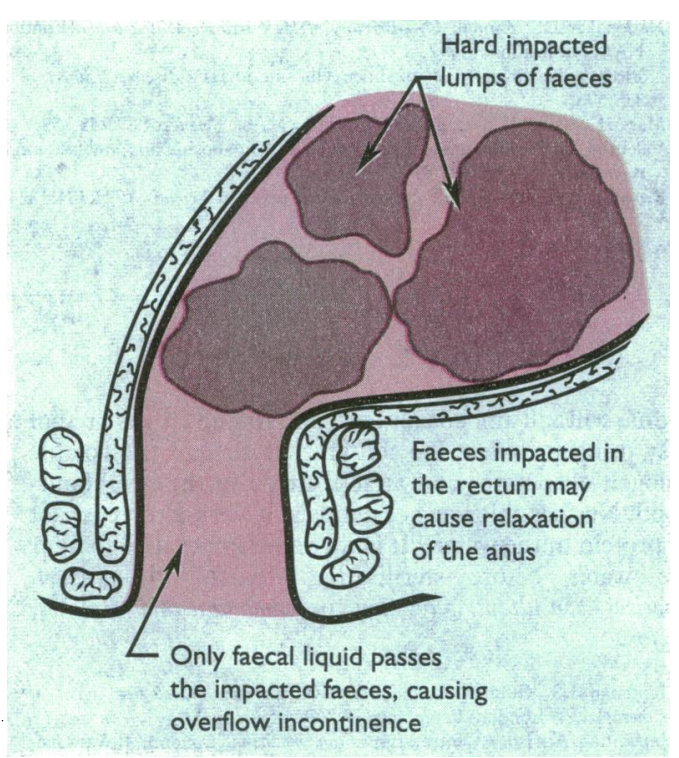

Faecal incontinence can be defined as the uncontrolled passage of stool through the rectum. This may be either because of a lack of awareness of the need to defecate caused by malfunction of the central or peripheral nervous system, or by an inability to get to a lavatory in time, which may be due to rapid transit through the bowel (for example, diarrhoea), poor rectal capacity (for example, chronic proctitis), or poor anal sphincter activity (for example, that caused by rectal prolapse or after obstetric sphincter injury or surgery for anal fistula). In many such cases treatment of the underlying condition cures the incontinence.

Faecal incontinence in elderly people is commonly associated with an impacted rectum and alleviated in most by disimpaction. Recent work has suggested that both the faecal impaction and incontinence are due to a local sensory deficit.

The key to success is to keep the stool as formed as possible and to initiate defecation with suppositories on a regular basis.

This approach can also be successful in the management of patients with multiple sclerosis, whose pelvic floor and sphincter mechanism is neither good enough to allow easy defecation of a solid stool nor strong enough to prevent incontinence of a loose stool.

There remains a considerable group of patients who are otherwise fit and well whose faecal incontinence is apparently of unknown aetiology. Such patients are severely incapacitated by their symptoms and yet many can be cured. There is accumulating evidence that many of these patients have suffered damage to the pudendal nerve. 

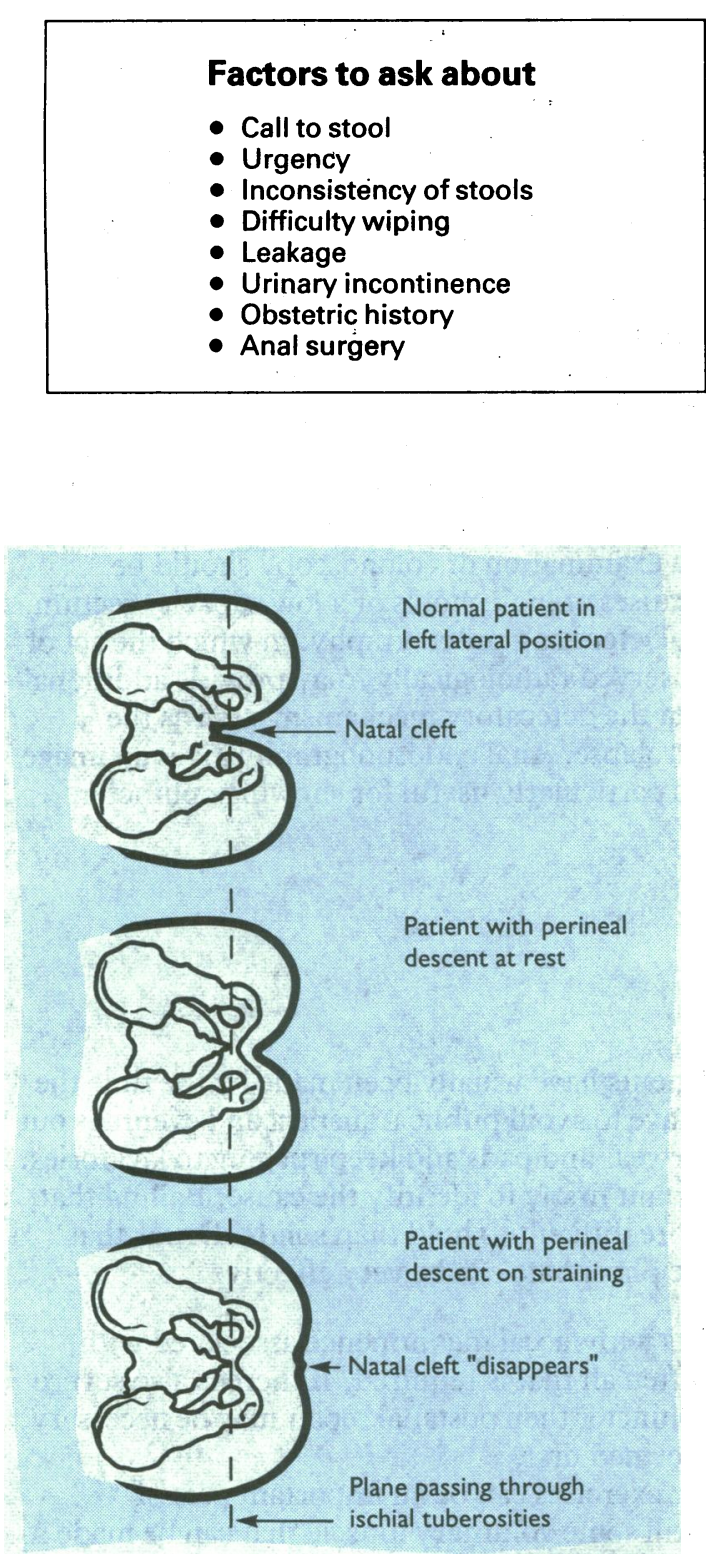

Weakness of the muscles of the pelvic floor is disclosed by a "descending perineum."

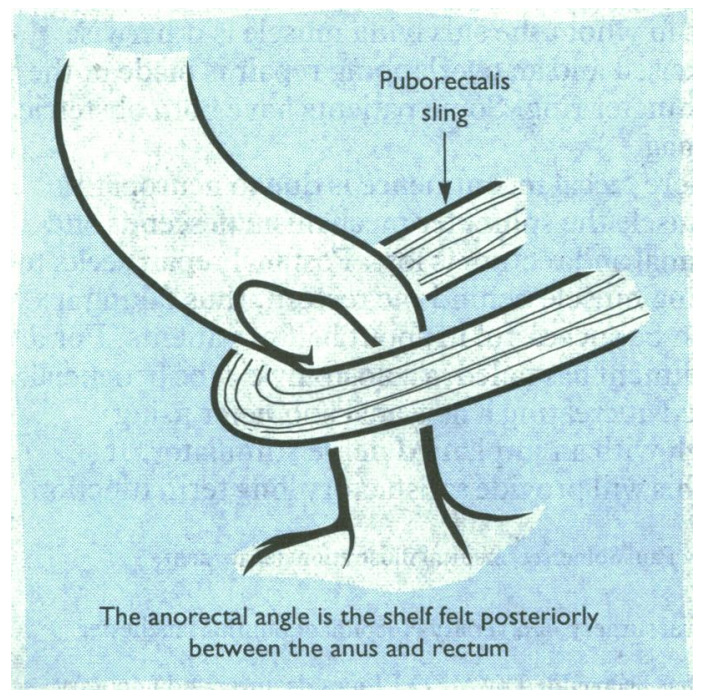

Position of anorectal angle.
People do not like to talk about defecation. Consequently, patients with faecal incontinence are embarrassed about it and tend to suffer with their symptoms for many years. It is important to ask the right questions - for example, about the nature of the call to stool - which is often altered or lost in patients with neuropathic incontinence. Urgency of defecation, with often only a few minutes' warning, when stools are solid suggests voluntary muscle weakness. Difficulty in wiping the anus indicates a lax sphincter or prolapse. Leakage between episodes of defecation-initially for flatus but eventually for solid stools - indicates low sphincter tone at rest (RT) and usually internal sphincter deficiency. Stress incontinence on coughing, bending, or walking suggests voluntary muscle weakness.

The majority of patients with idiopathic faecal incontinence are women and many have had one or more difficult deliveries. In most cases there is evidence of pudendal neuropathy. About half of these patients also have a history of urinary incontinence. The aetiology is probably the same as many women with urinary incontinence alone have evidence of subclinical damage to the innervation of the anal sphincters. A history of anal surgery, particularly anal dilatation, which can damage the internal sphincter, and surgery for a fistula may be important.

\section{Observation and digital examination}

In conjunction with the history, anal examination with the eye and the index finger will often lead to diagnosis.

Perianal soiling should alert the doctor to the possibility of faecal incontinence. Gaping of the anus at rest or on perianal traction means a low sphincter tone at rest and therefore poor internal sphincter function. Descent of the anal verge below the level of the ischial tuberosities suggests muscle weakness of the pelvic floor. Straining down by the patient may accentuate this or may reveal a rectal prolapse. Scars may indicate underlying muscle division.
Digital examination is obligatory. If inserting the gloved finger is easy the sphincter tone at rest is low-that is, the internal sphincter tone is poor. Feel the anorectal angle posteriorly. It should feel like a shelf. Ask the patient to squeeze tight and feel the difference in grip and the accentuation of the angle. Poor angle or poor squeeze means damaged voluntary muscles-either directly or due to pudendal neuropathy. Feel for faecal masses in the rectum and also for tumours, which may interfere with the anorectal mechanism. Endoscopy may reveal proctitis, which can reduce rectal capacity. 


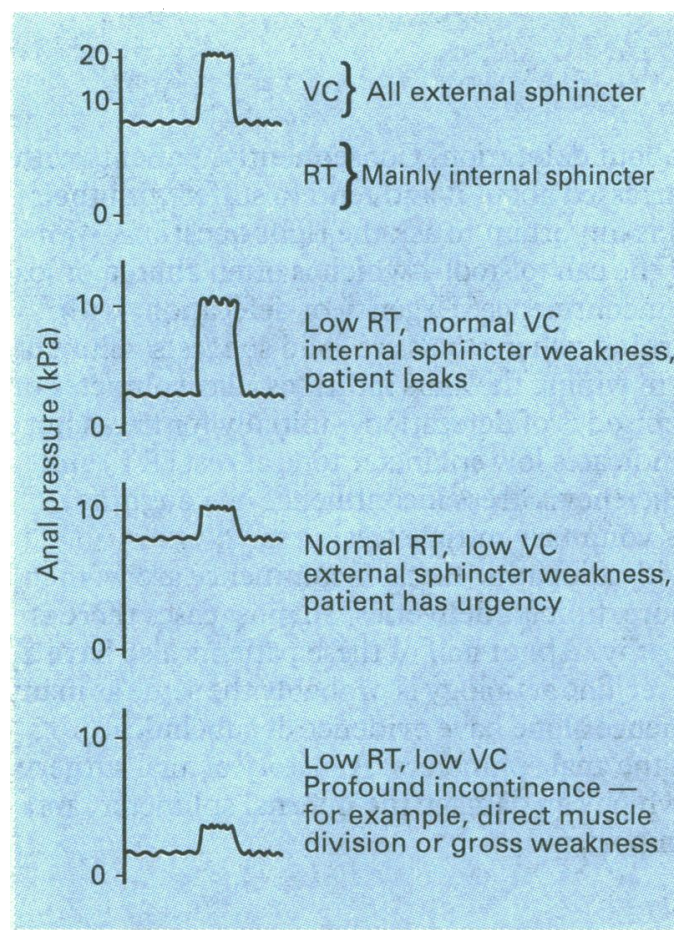

Anal manometry results showing the contributions of the internal and external sphincters to incontinence.

\section{Treatment}

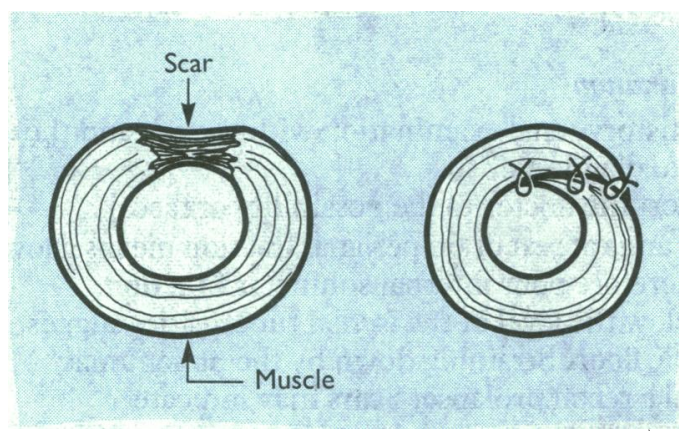

Overlapping sphincter repair gives good results in $85 \%$ of patients.

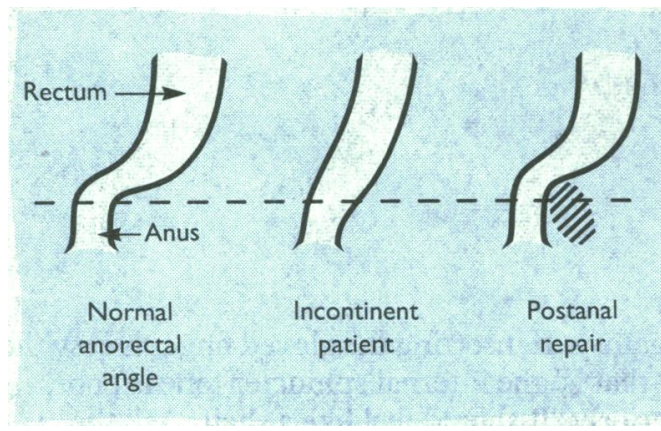

Postanal repair aims to recreate the anorectal angle by reinforcing the muscle posteriorly with suture material.

\section{Special tests}

What may have been suspected from the history and examination can be quantified by special tests.

Manometry-Manometry entails measuring the pressure exerted on a probe in the anal canal at rest (RT) and maximal voluntary contraction (VC). RT is mainly a function of the internal sphincter. VC is wholly a function of the external sphincter and voluntary muscles. To prevent incontinence, anal pressure must always exceed intrarectal pressure. Investigations using 24 hour ambulatory manometry have shown fluctuating anal pressures in some patients with faecal incontinence and pruritis ani.

Sensation-Patients with faecal incontinence often have an impaired ability to perceive rectal distension. This can be measured by inflating a balloon in the rectum. It may be possible to improve this ability by training.

Electromyography-Concentric needle electromyography can be used to identify the position of sphincter muscle in the traumatised anus but observation and digital examination are often as good. More sophisticated techniques are at present used only as research tools.

Radiology-Barium enema examination or colonoscopy should be performed to exclude other causes of loose stools or a low capacity rectum such as tumours or proctitis. Defecating proctography, in which the act of defecating barium paste is observed radiologically, may provide additional information on the disorder of the defecatory mechanism such as the presence of an occult rectal prolapse. Anal endosonography gives an image of the anal sphincter which is particularly useful for showing sphincter defects.

Physical and social adjustments have usually been made by the time the patient presents. They may have to avoid public transport and evenings out or always carry a bag of underwear and pads and keep rushing to lavatories. As in all treatment it is important firstly to identify the cause. Failing that, remember that liquids are more difficult to hold than solids. Drugs that solidify stools such as codeine phosphate can be very effective.

Rectal prolapse - In patients with faecal incontinence associated with rectal prolapse rectopexy is often all that is required. If there is also severe neuropathic damage to the sphincter then postanal repair may be necessary, but rectopexy should be performed first.

Physiotherapy-Pelvic floor exercises can be an important part of treatment provided there is still some voluntary muscle that can be made to work. Some patients have benefited from using muscle stimulating devices for a period of some months. This works best where there is good muscle bulk.

Trauma-For patients with faecal incontinence after direct obstetric or surgical trauma to the muscle, sphincter repair can be curative in about $85 \%$, particularly in patients in whom the surviving muscle is otherwise normal. The scar tissue is excised and an overlapping repair is made of the muscle ends to recreate a sphincter ring. Some patients have both obstetric trauma and neuropathic damage.

Neuropathic damage - Where faecal incontinence is due to neuropathic damage to the pelvic floor muscle the sphincter mechanism descends and the angle between the anal canal and rectum is lost. Postanal repair seeks to recreate this angle by opposing muscle behind the rectum, thus taking it upwards and forwards. It can be successful in about half of patients. For a few patients, when other treatment has failed, a colostomy can be beneficial.

Current research is directed at creating a new anal sphincter using gracilis muscle from the thigh with an implanted nerve stimulator. It remains to be seen whether this will provide satisfactory long term function.

The line drawings were prepared by Paul Somerset, medical illustration department, Wythenshawe Hospital, Manchester.

Mr E S Kiff is consultant in general surgery, University Hospital of South Manchester.

The ABC of Colorectal Diseases has been edited by Mr D J Jones, lecturer and honorary registrar, and Professor M H Irving, department of general surgery, Hope Hospital, Salford. 